\title{
Adubação nitrogenada na produção de mudas de mogno (Swietenia macrophylla King)
}

\author{
Carlos Alberto Franco TUCCI ${ }^{1}$, Hedinaldo Narciso LIMA², Josimar Ferreira LESSA ${ }^{3}$ \\ RESUMO \\ Estudos sobre aspectos silviculturais de espécies florestais nativas da Amazônia são escassos, principalmente aqueles que visam a \\ identificar técnicas de cultivo para a produção de mudas. O trabalho foi realizado objetivando avaliar efeitos de doses crescentes \\ de $\mathrm{N}$ no desenvolvimento de mudas de mogno. $\mathrm{O}$ estudo consistiu de sete tratamentos, correspondentes à doses crescentes e

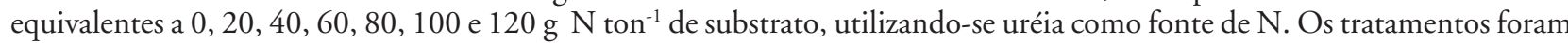 \\ distribuídos em delineamento experimental de blocos casualizados, com oito repetiçôes. Noventa dias após a repicagem foram \\ avaliadas as seguintes características: altura de planta, diâmetro do caule, matéria seca das raízes, do caule, das folhas e total. \\ Também se avaliou o conteúdo de $\mathrm{N}, \mathrm{P}, \mathrm{K}, \mathrm{Ca}$ e $\mathrm{Mg}$ nas folhas. Os resultados mostraram que a dose de máxima eficiência \\ física correspondeu a $61,5 \mathrm{~g} \mathrm{~N}$ ton ${ }^{-1}$ de substrato; o nível crítico de $\mathrm{N}$ para a parte aérea é de $27 \mathrm{~g} \mathrm{~kg}^{-1}$ e a dose para atingí- \\ lo de $57,5 \mathrm{~g} \mathrm{~N}^{-1}$ ton $^{-1}$ de substrato. Observou-se também que na dose de $120 \mathrm{~g} \mathrm{~N}^{-1}$ houve efeito negativo da adubação \\ nitrogenada sobre as características de crescimento avaliadas. Estes resultados indicam que as características de desenvolvimento \\ e de acumulação de nutrientes pelas mudas de mogno foram influenciadas positivamente por doses crescentes de $\mathrm{N}$, contudo \\ na dose máxima utilizada neste estudo $120 \mathrm{~g} \mathrm{~N}$ ton $^{-1}$, houve efeito negativo; a adubação nitrogenada resultou em aumento \\ no conteúdo de $\mathrm{N}, \mathrm{P}$ e Ca nas folhas; a dose de $\mathrm{N}$ recomendada para a produção de mudas de mogno, em substratos com \\ características semelhantes ao empregado neste estudo, é de $57,5 \mathrm{~g} \mathrm{~N}$ ton ${ }^{-1}$ de substrato.
}

PALAVRAS-CHAVE: Nitrogênio, Espécies florestais da Amazônia, Mogno.

\section{Nitrogen fertilization in the production of seedlings of mahogany (Swietenia macrophylla King)}

\begin{abstract}
Studies on silvicultural aspects of native forest species of the Amazon Rainforest are scarce, mainly those that seek to establish cultivation techniques for production of seedlings. The current work had the objective of evaluating the effects of growing levels of $\mathrm{N}$ in the development of mahogany seedlings. The treatments were seven growing doses that were equivalent to the application of: $0 ; 20 ; 40 ; 80 ; 100$ and $120 \mathrm{~g} \mathrm{~N}^{-1}$ of the substratum. The source of $\mathrm{N}$ was urea. The experimental design was randomized blocks with eight repetitions. Evaluations were accomplished after 90 days of transplanting where it was observed answers to the growing doses of $\mathrm{N}$ for the diameter of stem, dry matter of the leaves and for total content of $\mathrm{N}, \mathrm{P}$, $\mathrm{K}, \mathrm{Ca}$ and $\mathrm{Mg}$ of the leaves. The recommendation of nitrogen fertilization for the production of mahogany seedlings is of $57,5 \mathrm{~g} \mathrm{~N}^{-1}$ of the substratum as a first approach.
\end{abstract}

KEY WORDS: Nitrogen, forest species of Amazon Rainforest, mahogany.

'Departamento de Engenharia Agrícola e Solos da Universidade Federal do Amazonas. Av. Gel. Rodrigo Otávio Jordão Ramos, 3000 - Bloco A. 69070-000 -Manaus, AM. e-mail: ctucci@ufam.edu.br

2Professor Associado do Departamento de Engenharia Agrícola e Solos da Universidade Federal do Amazonas. e-mail: hedinaldo@ufam.edu.br

3Universidade Federal do Amazonas, bolsista FAPEAM. e-mail: josimarflessa@ufam.edu.br 


\section{INTRODUÇÃO}

O mogno (Swietenia macrophylla King), que pertence à família Meliaceae, é uma espécie arbórea semidecídua ou decídua comumente encontrada nas florestas de terra firme da Amazônia brasileira.

A demanda pela madeira do mogno e o consequente extrativismo cresceram significativamente nos anos mais recentes. Enquanto os estoques permaneceram limitados à população natural e a sua regeneração.

Mais recentemente, devido ao incremento da demanda, aos problemas ambientais decorrentes do extrativismo desordenado e ao valor comercial da madeira, o cultivo dessa espécie tem aumentado significativamente.

Todavia, grande parte dos cultivos isolados ou consorciados fracassou por problemas relativos às condiçōes de cultivo, entre os quais a qualidade das mudas. A qualidade de mudas é definida por características vegetativas, além do potencial hídrico e estado nutricional das mudas. Todas essas características são influenciadas pela qualidade do substrato e das sementes.

A utilização de terra do subsolo para a produção de mudas em viveiro tornou-se uma prática usual com a vantagem de não apresentar a incidência de doenças. Por outro lado, o material do subsolo, de modo geral, apresenta baixa disponibilidade de nutrientes e acidez elevada, necessitando de fertilização e correção.

As limitaçōes da fertilidade do substrato têm sido consideradas um dos fatores responsáveis por perdas de mudas e causa de elevada mortalidade das plantas por ocasião do plantio definitivo no campo.

Os teores de nutrientes no substrato, principalmente nitrogênio, fósforo e potássio têm grande influência na qualidade das mudas produzidas e no desenvolvimento das mesmas em campo. Respostas positivas à correção e fertilização, especialmente nitrogenada, do substrato sobre características do crescimento de mudas de mogno têm sido observadas por alguns autores, entre os quais Barros (2001). Furtini Neto et al. (2000), Silva et al., (2007) e Tucci et al., (2001) também relataram efeitos positivos da adubação nitrogenada sobre o crescimento de outras espécies arbóreas.

O presente trabalho teve como objetivo avaliar os efeitos de doses crescentes de $\mathrm{N}$ no crescimento de mudas de mogno, bem como estimar a dose para máxima eficiência econômica e o nível crítico de $\mathrm{N}$ na parte aérea destas.

\section{MATERIAL E MÉTODOS}

Utilizou-se como substrato para a produção das mudas terra da camada subsuperficial de um Latossolo Amarelo Distrófico típico, coletado no Campus Universitário da
Universidade Federal do Amazonas, em Manaus-AM, nas seguintes coordenadas UTM são 21 M 016894; 9657166.

Amostras do material coletado foram secas ao ar, peneiradas, em peneira com abertura de malha de $2 \mathrm{~mm}$, e em seguida submetidas a análises para caracterização da fertilidade química e da composição granulométrica.

Após a caracterização química e a determinação da composição granulométrica calculou-se a necessidade de calagem, de acordo Catani e Alonso (1969). A correção do substrato foi feita utilizando-se uma mistura de carbonato de cálcio mais carbonato de magnésio, na proporção 4:1, respectivamente. Depois da aplicação do corretivo, e homogeneização da mistura, esta foi incubada durante 30 dias, para a solubilização e reação do corretivo com o substrato.

Ao final do período de incubação, foi realizada a fertilização do substrato, tomando por base suas características químicas, utilizando superfosfato triplo, cloreto de potássio e gesso em doses equivalentes a 150,100 e $15 \mathrm{~g}^{-1}$ ton $^{-1}$ de substrato de $\mathrm{P}_{2} \mathrm{O}_{5}, \mathrm{~K}_{2} \mathrm{O}$ e $\mathrm{S}$ respectivamente. Adicionalmente, aplicouse solução nutritiva contendo $\mathrm{B}, \mathrm{Fe}, \mathrm{Cu}, \mathrm{Mn}, \mathrm{Zn}$ e $\mathrm{Mo}$ em doses equivalentes a 1,$0 ; 10,0 ; 1,5 ; 5,0 ; 5,0$ e $0,1 \mathrm{~g} \mathrm{ton}^{-1} \mathrm{de}$ substrato, respectivamente.

As mudas utilizadas no experimento foram produzidas a partir de sementes, tratadas com benlate a $1 \%$, e semeadas em substrato de areia lavada. Trinta dias após a germinação, as plântulas foram selecionadas em função da uniformidade em altura, coloração e desenvolvimento do sistema radicular e transplantadas para sacos de polietileno com capacidade de $4 \mathrm{~kg}$ de substrato.

Os tratamentos consistiram de doses crescentes de $\mathrm{N}$ equivalentes a $0 ; 20 ; 40 ; 60 ; 80 ; 100$ e $120 \mathrm{~g} \mathrm{~N}^{-1}$ de $^{-1}$ substrato. A fonte de $\mathrm{N}$ utilizada foi a uréia. Os tratamentos foram distribuídos em delineamento experimental de blocos casualizados com oito repetições.

O tempo de duração do ensaio, após o transplantio foi de 90 dias. Durante esse período foi realizada irrigação diária. A quantidade de água utilizada na irrigação foi correspondente a $30 \%$ do peso do solo.

Ao final do período de ensaio foram avaliadas as seguintes características: altura da planta (AP), número de folhas (NF), diâmetro do caule (DC), matéria seca de raiz (MSR), caule (MSC), folhas (MSF), parte aérea (MPA) obtida pela soma de MSC + MSF, e total (MST), relação de matéria seca de parte aérea/matéria seca da raiz (MPA/MSR). Os teores de $\mathrm{N}, \mathrm{P}, \mathrm{K}, \mathrm{Ca}$ e $\mathrm{Mg}$ na parte aérea das folhas foram avaliados de acordo com o procedimento descrito por Malavolta $e t$ al. (1997). As estimativas dos conteúdos de N (CN), P (CP), K (CK), $\mathrm{Ca}(\mathrm{CCa})$ e $\mathrm{Mg}(\mathrm{CMg})$ na parte aérea das folhas foram obtidas multiplicando-se o teor de cada elemento matéria seca das folhas. 
Foram ajustados modelos de regressão entre as características avaliadas como variável dependente das doses de nitrogênio aplicadas. A dose para máxima eficiência física (MEF) foi obtida derivando-se a equação ajustada para matéria seca total e a dose para máxima eficiência econômica (MEE) foi estimada considerando-se $90 \%$ da produção com a dose para a MEF. O nível crítico de $\mathrm{N}$ na parte aérea foi estimado substituindo-se a dose para MEE na equação de conteúdo de $\mathrm{N}$ na parte aérea.

\section{RESULTADOS E DISCUSSÃO}

\section{CARACTERIZAÇÃO DO SUBSTRATO}

$\mathrm{O}$ valor do $\mathrm{pH}$ em água do material utilizado como substrato foi de 4,6, o que caracteriza um material fortemente ácido, de acordo com as classes de reação do solo, estabelecidas pelo Sistema Brasileiro de Classificação de Solos (SisBCS) (Embrapa, 2006). O teor elevado de $\mathrm{Al}^{3+}\left(1,75 \mathrm{cmol}_{\mathrm{c}} \mathrm{kg}^{-1}\right)$, associado a baixos valores de soma de bases $\left(0,12 \mathrm{cmol}_{\mathrm{c}} \mathrm{kg}^{-1}\right)$, resultou em saturação por alumínio muito elevada e muito baixa saturação por bases. Destaca-se também o baixo teor de P (Tabela 1). Esses resultados confirmam a baixa fertilidade natural e elevadas acidez e saturação por alumínio, geralmente observadas nos Latossolos da Amazônia (Rodrigues, 1996; Schaefer et al., 2000).

\section{EFEITOS DOS TRATAMENTOS SOBRE AS VARIÁVEIS ANALISADAS}

Os resultados deste estudo mostram respostas positivas às doses de $\mathrm{N}$ das seguintes variáveis: diâmetro do caule, produção de matéria seca das folhas, matéria seca total e no conteúdo de N, P e Ca nas folhas. Efeitos positivos da adubação nitrogenada sobre características de crescimento de espécies florestais foram observados por vários autores Dias et al., (1992); Macedo et al., (1999); Pereira, (1998); Tucci et al., (2001).

As respostas de matéria seca das folhas, matéria seca total, conteúdo de $\mathrm{P}$ e de Ca nas folhas apresentaram melhor ajuste ao modelo quadrático (Figuras 1 e 2). No entanto, o conteúdo de $\mathrm{N}$ apresentou resposta mais bem ajustada ao modelo linear (Figura 2).

Por outro lado, não se ajustaram modelos de regressão para as características diâmetro de caule, matéria seca da raiz e do

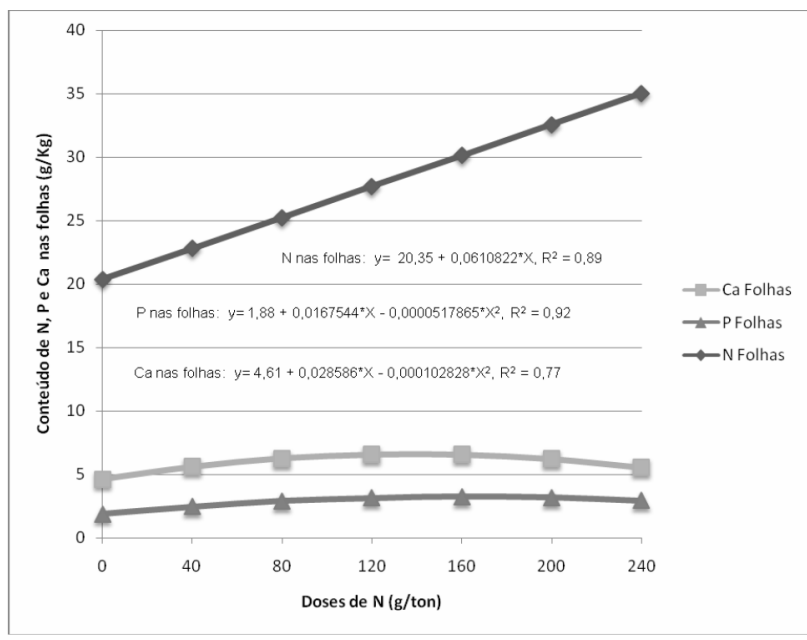

Figura 1 - Equações de regressão para matéria seca da folha (MSF) e matéria seca total (MST) como variável dependente das doses de nitrogênio aplicadas.

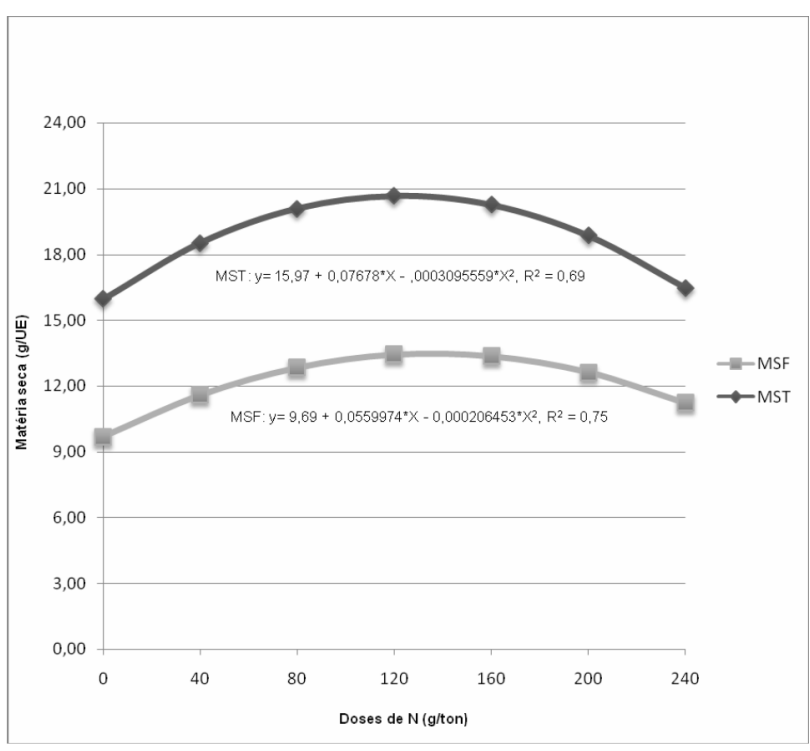

$(\mathrm{g} / \mathrm{UE})=$ gramas/unidade experimental

Figura 2 - Equações de regressão para matéria seca da folha (MSF) e matéria seca total (MST) como variável dependente das doses de nitrogênio aplicadas.

Tabela 1 - Características químicas e classe textural da terra utilizada como substrato.

\begin{tabular}{|c|c|c|c|c|c|c|c|c|c|c|c|}
\hline $\mathrm{pH}$ & $\mathrm{Al}^{3+}$ & $\mathrm{H}+\mathrm{Al}$ & $\mathrm{Ca}^{2+}+\mathrm{Mg}^{2+}$ & SB & $T$ & $\mathrm{t}$ & $P$ & K & V & $\mathrm{m}$ & \multirow{2}{*}{ Classe textural } \\
\hline $\mathrm{H}_{2} \mathrm{O}$ & & & $\mathrm{cmol}_{\mathrm{c}} \mathrm{kg}$ & & & & \multicolumn{2}{|c|}{$\mathrm{mg} \mathrm{kg}^{-1}$} & $\%$ & & \\
\hline 4,6 & 1,75 & 8,59 & 0,10 & 0,12 & 8,71 & 1,87 & 1 & 8 & 1,4 & 94 & Argila \\
\hline
\end{tabular}


caule. Por isso, os resultados dos tratamentos foram analisados por comparação das médias dos tratamentos (Tabela 2).

Esses resultados mostram que não houve efeitos da adubação nitrogenada no diâmetro de caule, em comparação a sua ausência. Por outro lado, observaram-se efeitos negativos da adubação nitrogenada na maior dose avaliada $\left(240 \mathrm{~g} \mathrm{~N}_{\text {ton }^{-1}}\right)$, sendo o crescimento do diâmetro do caule, estatisticamente, inferior ao crescimento observado na dose de $80 \mathrm{~g} \mathrm{~N}$ ton $^{-1}$ (Tabela 2). Esses resultados diferem daqueles observados por Barros (2001), que estudando efeitos de adubação com NPK sobre o crescimento de mudas de mogno, observou crescimento do diâmetro do caule de quase $100 \%$, em relação a ausência de adubação.

$\mathrm{O}$ resultado da ANOVA apresentou $\mathrm{F}$ significativo, ao nível de 5\% de probabilidade, para a característica matéria seca $\mathrm{da}$ raiz, indicando que deveria haver pelo menos um contraste não nulo entre as médias dos tratamentos. Neste caso, embora, não se tenha observado diferença entre o tratamento sem adubação nitrogenada e os demais tratamentos, observou-se diferença significativa entre a média da dose de $160 \mathrm{~g} \mathrm{ton}^{-1} \mathrm{e}$ a dose $240 \mathrm{~g}^{-1}{ }^{-1}$ (Tabela 2).

Como destacado anteriormente, o uso da dose máxima $\left(240 \mathrm{~g} \mathrm{ton}^{-1}\right)$, neste estudo, provocou efeito negativo sobre as variáveis diâmetro do caule e matéria seca da raiz. Com a redução da matéria seca das raízes observa-se aumento de relação parte aérea/raiz (Tabela 2), o que pode ocasionar efeito negativo para a qualidade das mudas. Todavia, sugere-se que novas pesquisas devem ser conduzidas para se avaliar melhor os efeitos do $\mathrm{N}$ sobre essas características e outras características de crescimento das mudas de mogno.

Destaca-se também que não se observaram efeitos das doses crescentes de $\mathrm{N}$ na altura das plantas, número de folhas, relação parte aérea/raiz; conteúdos foliares de potássio e magnésio. Estes resultados foram opostos aos encontrados por Barros (2001).

\section{DETERMINAÇÃO DA DOSE DE MÁXIMA EFICIÊNCIA}

A dose para máxima eficiência física foi estimada em $61,5 \mathrm{~g} \mathrm{~N}$ ton $^{-1}$ e para máxima eficiência econômica, aproximadamente, $57,5 \mathrm{~g} \mathrm{~N}^{-n^{-1}}$ de substrato. O nível crítico de $\mathrm{N}$ na parte aérea das folhas mogno foi estimado em $27 \mathrm{~g} \mathrm{~kg}^{-1}$. Silveira et al. (2000) verificou que $21,8 \mathrm{~g} \mathrm{~kg}^{-1} \mathrm{de}$ $\mathrm{N}$ na folha foi o teor do nutriente associado com a máxima produtividade de Eucaliptus grandis, e $21 \mathrm{~g} \mathrm{~kg}^{-1}$ de $\mathrm{N}$ como nível critico foliar, pode-se considerar que $E$. grandis e mogno apresentam valores de níveis críticos próximos.

A dose para atingir o nível crítico de $\mathrm{N}$ na parte aérea correspondeu à aplicação de $57,5 \mathrm{~g} \mathrm{de} \mathrm{N}$ ton $^{-1}$ de substrato. A maior produção de matéria seca de folha foi observada com a dose de $68 \mathrm{~g} \mathrm{~N}_{\text {ton }}{ }^{-1}$, que foi estimada derivando-se a equação ajustada para matéria seca de folhas como variável dependente das doses de nitrogênio aplicadas (Figura 2).

Observou-se correlação positiva e significativa entre doses de $\mathrm{N}$ aplicado x P acumulado $\left(\mathrm{r}=0,88^{*}\right)$ e doses de $\mathrm{N}$ x Ca acumulado $\left(\mathrm{r}=0,99^{*}\right)$ na matéria seca das folhas. Tal fato evidenciou que o $\mathrm{N}$ favoreceu a absorção e/ou utilização do P e do Ca pela planta. Pereira (1995), trabalhando com várias espécies florestais, observou que a adição de $\mathrm{N}$ promoveu maior acúmulo de $\mathrm{Ca}$ e de $\mathrm{P}$ nas plantas, sendo a forma amoniacal mais eficiente quando comparada à nítrica. Tal fato tem sido explicado pela competição entre íons pelos sítios carreadores (Hageman, 1984); para o jacarandá (Jacaranda minoseafolia) isto não ocorreu, evidenciado diferente comportamento dessa espécie.

Por outro lado, Renó (1994) verificou que a omissão de $\mathrm{N}$ resultou em menores teores de $\mathrm{P}, \mathrm{K}, \mathrm{Mg}, \mathrm{Mn}, \mathrm{Cu}$ e $\mathrm{Zn}$ na parte aérea de cedro, indicando a influência do teor de $\mathrm{N}$ no

Tabela 2 - Valores médios ${ }^{(1)}$ para diâmetro do caule (DC), matéria seca de raízes (MSR), caule (MSC) e conteúdo de cálcio na parte aérea (CCa) e relação parte aérea/raiz (MPA/MSR)

\begin{tabular}{llll}
\hline Dose de $N$ & $\mathrm{DC}$ & $\begin{array}{l}\text { MSR } \\
\mathrm{gm} / \mathrm{UE}\end{array}$ & MPA/MSR \\
\hline 0 & $\mathrm{~mm}$ & $2,94 \mathrm{AB}$ & 5,5131 \\
40 & $8,02 \mathrm{AB}$ & $2,45 \mathrm{AB}$ & 6,2782 \\
80 & $7,21 \mathrm{AB}$ & $2,94 \mathrm{AB}$ & 6,4627 \\
120 & $8,18 \mathrm{~A}$ & $3,16 \mathrm{AB}$ & 5,3105 \\
160 & $7,36 \mathrm{AB}$ & $3,63 \mathrm{~A}$ & 5,3892 \\
200 & $7,80 \mathrm{AB}$ & $2,96 \mathrm{AB}$ & 5,4285 \\
240 & $7,19 \mathrm{AB}$ & $1,97 \mathrm{~B}$ & 7,5990 \\
\hline
\end{tabular}

${ }^{(1)}$ Médias seguidas da mesma letra não diferem pelo teste de Tukey a $5 \%$ de probabilidade. ${ }^{(2)}(\mathrm{g} / \mathrm{UE})=$ gramas/unidade experimental. 
substrato sobre a absorção e metabolismo de outros nutrientes. Neves et al. (1990) mencionam que na grande maioria dos substratos utilizados na produção de mudas de eucalipto a ausência de adubação nitrogenada pode resultar em respostas limitadas à adição de $\mathrm{P}$ e $\mathrm{S}$.

\section{CONCLUSÕES}

A maioria das características de crescimento e a acumulação de nutrientes pelas mudas de mogno foram influenciadas positivamente pela adubação nitrogenada.

A dose máxima empregada neste estudo $\left(240 \mathrm{~g} \mathrm{~N}\right.$ ton $^{-1} \mathrm{de}$ substrato) causou efeito negativo sobre o diâmetro do caule e a matéria seca da parte aérea, prejudicando a qualidade das mudas.

A dose de $\mathrm{N}$ recomendada para a produção de mudas de mogno, utilizando-se substrato com características semelhantes é de $57,5 \mathrm{~kg} \mathrm{~N}^{-1}{ }^{-1}$ substrato e o nível crítico na parte aérea é de $27 \mathrm{~g} \mathrm{~kg}^{-1}$ de $\mathrm{N}$.

\section{BIBLIOGRAFIA CITADA}

Barros, J.G. 2001. Adubação e calagem para a formação de mudas de mogno (Swietenia macrophylla King). Dissertação de Mestrado, Universidade Federal do Amazonas, Manaus, Amazonas, $63 \mathrm{pp}$.

Catani, R.A.; Alonso, O. 1969. Avaliação da exigência de calcário do solo. Piracicaba. Anais da Escola Superior de Agricultura Luis de Queiroz, Piracicaba p.141-156.

Dias, L.E.; Jucksch, I.; Alvarez, V.H.V.; Franco, A.A.; Brienza Jr.S. 1992. Formação de mudas de táxi-branco (Scherolobium paniculatum Voguel): Resposta a nitrogênio, potássio e enxofre. Revista Arvore, 16(2): 135-143.

EMPRESA BRASILEIRA DE PESQUISA AGROPECUÁRIA. EMBRAPA. 2006. Sistema Brasileiro de Classificação de Solos. Rio de Janeiro, Centro Nacional de Pesquisa de Solo. 306pp.

Furtuni Neto, A.E.; Siqueira, J.O.; Curi, N.; Moreira, F.M.S. 2000. Fertilização em reflorestamento com espécies nativas. In.: Gonçalves, J.L. de M.; BENEDETTI, V. (Eds). Nutrição e fertilização florestal. Piracicaba. IPEF, p.351-383.

Hageman, R.H. 1984. Ammonium versus nitrate nutrituion of higher plants. In: Hauck, R.D., (ed.) Nitrogen in Crop Production. Madison. 804pp.

Macedo, R.L.G.; Santos Filho, R.N.; Venturin, N.; Pereira, J.A.A.; Gomes, J.E. 1999. Efeitos da simulação de adubação de plantio sobre o desenvolvimento de Colophyllum brasiliensis camb. (guanandi). Anais do Quinto Congresso e Exposição Internacional Sobre Floresta - FOREST 99. Curitiba.

Malavolta, E.; Vitti, G.C.; Oliveira, S.A. de. 1989. Avaliação do estado nutricional das plantas: princípios e aplicaçôes. Piracicaba. POTAFOS. 210pp.

Neves, J.C.L.; Gomes, J.M.; Novais, R.F. de. 1990. Fertilização mineral de mudas de eucalipto. In: Barros, N.F.; Novais, R.F de (Eds). Relação solo-eucalipto. Viçosa. Folha de Viçosa. p.99126.

Pereira, L.A. 1998. Comportamento do paricá (Schizolobium amazonicum Ducke) em um sistema agroflorestal implantado em pastagem degradada. Manaus. Monografia, Universidade Federal do Amazonas, Manaus. 32pp.

Renó, N.B. 1994. Requerimentos nutricionais e resposta ao fósforo e fungo micorrizico em espécies arbóreas nativas no sudeste brasileiro. Dissertação de Mestrado, Escola Superior de Agricultura Luiz de Queiroz, Lavras. 62pp.

Rodrigues, T.E. Solos da Amazônia. In: O solo nos grandes domínios morfoclimáticos do Brasil e o desenvolvimento sustentado/ Editado por Victor Hugo Alvarez V., Luiz Eduardo F. Fontes, Maurício Paulo F. Fontes. Viçosa, MG: SBCS; UFV, DPS. p. 19-60. 1996.

Schaefer, C.E.G.R.; Lima, H.N.; Vale Júnior, J.F.; Mello, J.W.V. 2000. Uso dos solos e alteraçôes da paisagem na Amazônia: cenários e reflexôes. Bol. Mus. Para. Emílio Goeldi, sér. Ciênc. da Terra, 12:63-104.

Silva, A.R.; Tucci, C.A.F.; Lima, H.N.; Figueiredo, A.F. de. 2007. Doses crescentes de corretivo na formação de mudas de mogno (Swietenia macrophylla King). Acta Amazonica, 37(2): 195200.

Silveira, L.V.A.; Higashi, E.N.; Gonçalves, A.N.; Moreira, A. 2000. Avaliação do estado nutricional do Eucaliptus: diagnose visual, foliar e suas interpretaçôes. In.: Gonçalves, J.L. de M.; Benedetti, V. (Eds). Nutrição e fertilização florestal. Piracicaba. IPEF. p. $80-104$

Tucci, C.A.F.; Hara, F.A. dos S.; Freitas, R.O. de. 2001. Adubação e calagem para a formação de mudas de sumaúma (Ceiba pentandra L. Gaertn). Rev. da Universidade Federal do Amazonas, Manaus. Série Ciências Agrárias, 10(1/2).

Recebido em 23/01/2008

Aceito em 27/08/2008 
